\title{
Corrigendum: "Parameter Sensitivities of the Dual-Localization Approach in the Local Ensemble Transform Kalman Filter"
}

\author{
Keiichi Kondo ${ }^{1}$, Takemasa Miyoshi ${ }^{1,2,3}$ and H. L. Tanaka ${ }^{4}$ \\ ${ }^{1}$ RIKEN Advanced Institute for Computational Science, Kobe, Japan \\ ${ }^{2}$ Department of Atmospheric and Oceanic Science, University of Maryland, College Park, Maryland, USA \\ ${ }^{3}$ Japan Agency for Marine-Earth Science and Technology, Yokohama, Japan \\ ${ }^{4}$ Center for Computational Sciences, University of Tsukuba, Tsukuba, Japan
}

(Citation: Kondo, K., T. Miyoshi, and H. L. Tanaka, 2015: Corrigendum: "Parameter sensitivities of the dual-localization approach in the local ensemble transform Kalman filter". SOLA, 11, c3, doi:10.2151/sola.2015-017.)

Kondo et al. (2013) presented the root mean square errors (RMSE) in their Figs. 4 and 5. However, it turned out that the mean absolute errors (MAE) were shown instead. The descriptions of Kondo et al. (2013) will be consistent if we replace all "RMSE" to be "MAE" for their Figs. 4 and 5 and the related descriptions in Sections 4 and 5. The conclusions do not change due to this error.

\section{References}

Kondo, K., T. Miyoshi, and H. L. Tanaka, 2013. Parameter sensitivities of the dual-localization approach in the local ensemble transform Kalman filter. SOLA, 9, 174-178.

Corresponding author: Keiichi Kondo, Data Assimilation Research Team, RIKEN Advanced Institute for Computational Science, 7-1-26 Minatojima-minami-machi, Chuo-ku, Kobe, Hyogo, 650-0047, Japan. E-mail: keiichi.kondo@riken.jp. (C)2015, the Meteorological Society of Japan. 